\title{
246 GHz Digitally Stitched Coherent Receiver
}

\author{
Kai Shi, Eric Sillekens and Benn C. Thomsen \\ Department of Electronic and Electrical Engineering, University College London, London, WC1E 7JE, UK \\ k.shi@ucl.ac.uk
}

\begin{abstract}
Phase estimation and 4×2 MIMO equalization techniques are experimentally compared for digital frequency stitching in ultra-wideband coherent reception, using time multiplexing and a single conventional dual polarization coherent receiver, to simultaneously detect a $5 \times 46 \mathrm{GBaud}$ super-channel.

OCIS codes: (060.1660) Coherent communications; (070.7145) Ultrafast processing
\end{abstract}

\section{Introduction}

Ultra wide-band coherent receivers are critical for high capacity transmission as it enables nonlinear interference mitigation inherent in optical fiber transmission systems [1]. However, the bandwidth of optical coherent receivers is limited by high-speed electronics and photodiodes to around $120 \mathrm{GHz}$. A frequency-translating hybrid architecture is commonly used in wide-band analog-to-digital converters [2], and this technique has been demonstrated in the optical domain to detect a 214 GBbaud single carrier signal and also a $1.2 \mathrm{~Tb} / \mathrm{s}$ super-channel using multiple synchronized low speed coherent receivers $[1,3]$. The key advantage of this technique is that it allows capture of optical symbol rates beyond the electronic limits. In this technique, each of the spectral slices are detected coherently in order to calibrate for the phase differences between each spectral slice before the detected signals are digitally recombined.

Here we show that for experimental systems, using offline processing, which only capture a short block of the signal, the multiple synchronized coherent receivers structure $[1,3]$ can be simplified by using a parallel to serial conversion, similar to those used in mode division multiplexing systems, where a single coherent receiver can be used to detect multiple parallel channels [4]. Fiber delay lines are introduced to create parallel copies of the signal block which are then serialized before detection in a single coherent receiver at different time slots. In this paper, we demonstrate an optical time multiplexed frequency stitched (FS) architecture using only one single coherent receiver. We compare the phase estimation stitching approach [1] with a $4 \times 2$ multiple-input multiple-output MIMO equalizer structure. Frequency banded MIMO equalization has previously been used for adaptive channel separation of frequency overlapped channels [5], here we use it to combine the frequency sub-bands, alleviating the accuracy of the phase calibration process for the spectral stitching. We demonstrate the reception of a 60 GBaud DP Nyquist signal using a 40 GSamples/s (GS/s) ADCs with an electrical bandwidth of approximately $16 \mathrm{GHz}$ and simultaneous reception of a $5 \times 46$ Gbaud $(246 \mathrm{GHz}$ ) super-channel by using a single $63 \mathrm{GHz}$ electrical bandwidth coherent receiver.

\section{Time multiplexed FS receiver}

The experimental setup of the FS receiver is shown in Fig.1 (a). In order to frequency-translate the wideband signal, a set of phase locked local oscillators (LOs) is required to coherently downconvert each sub-band of the signal to baseband with a certain phase offset. In our experiment, the phase locked optical frequency comb is generated by an intensity modulator followed by a phase modulator [6], both of which are driven by a sinusoidal signal at a frequency according to the bandwidth of the signal and the number of required spectral slices. A frequency de-interleaver is then used to filter out the comb lines at double the comb spacing. This alleviates the requirement of the resolution of the

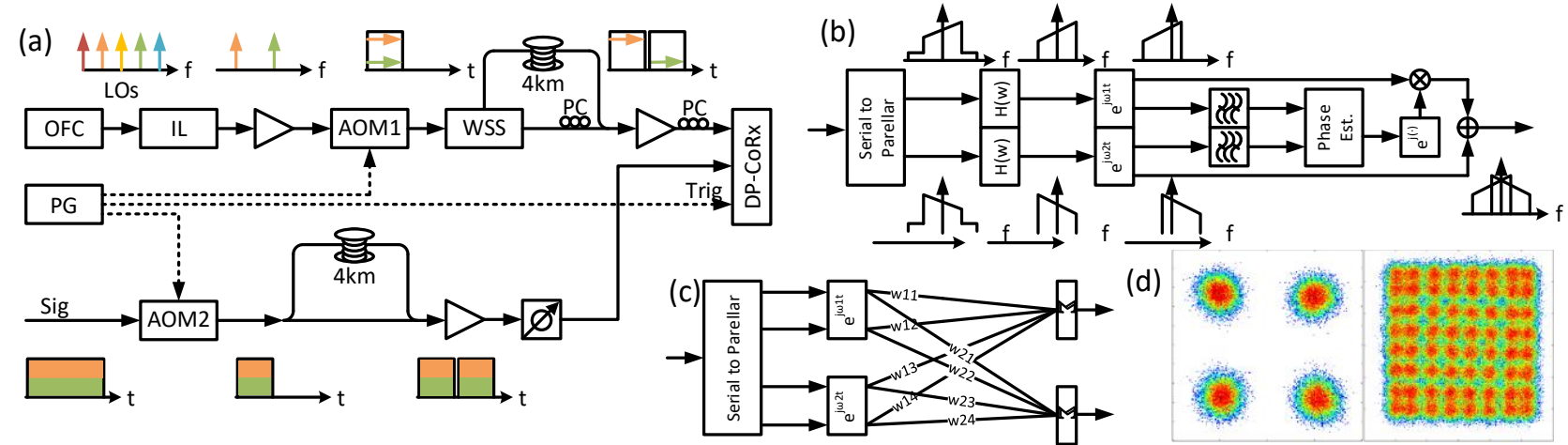

Fig. 1. (a) Setup diagram of the FS receiver. OFC: optical frequency comb, IL: frequency interleaver, AOM: acousto-optic modulator, WSS: wavelength selective switch, PG: pattern generator, PC: polarization controller. (b) Frequency stitching algorithms using phase correction and (c) $4 \times 2$ MIMO equalizer. (d) Constellation diagram after the $4 \times 2$ MIMO equalizer with an OSNR of $34 \mathrm{~dB}$ for 60 GBaud QPSK and 46 GBaud 64 QAM signals. 

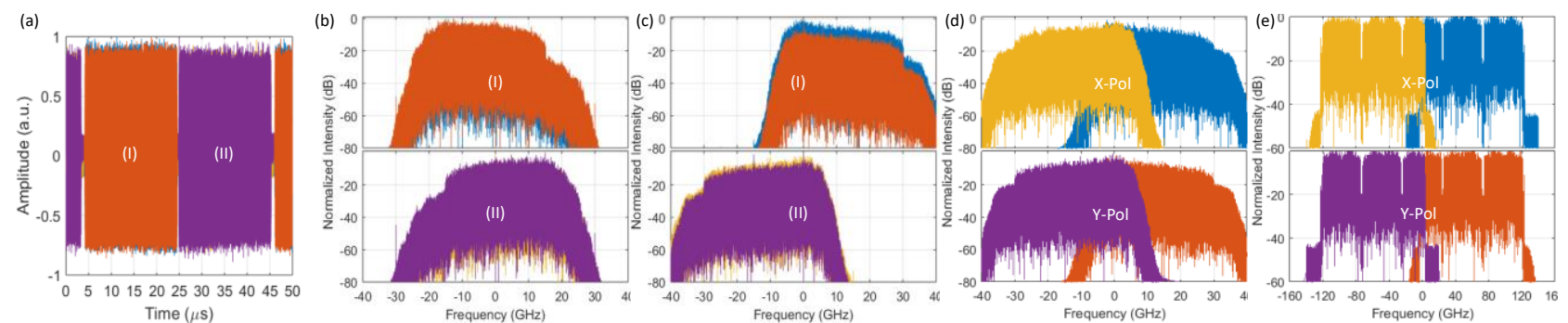

Fig. 2. (a) Captured waveforms of the $x$ (blue and yellow) and $y$ (red and purple) polarizations. The signals in the first (I) and second (II) time slots correspond to the frequency-translated higher and lower half of the $60 \mathrm{Gbaud}$ signal. Electrical spectra of (b) the filtered baseband signal, (c) after frequency translation of $\pm 15 \mathrm{GHz}$, and after phase correction and stitching of (d) a single 60 Gbaud and (e) $5 \times 46$ Gbaud signals.

wavelength selective switch (WSS) $(\sim 1 \mathrm{GHz})$, which is used to select the required LO lines. An acousto-optic modulator (AOM1) is used as a shutter to create an LO signal of the same duration as the signal block that we wish to capture. This is followed by the WSS to select the desired comb lines and demultiplex them into two different delays. The difference of the delay is chosen to be slightly larger than the signal block duration so that we can distinguish the two packages detected by the receiver. In our setup, the shuttering periods of AOMs are set at $21 \mu \mathrm{s}$, which corresponds to a fiber delay length of approximately $4 \mathrm{~km}$. Similar shuttering and delay are also introduced to the received signal. However, the WSS is no longer required and the two copies of the wide band signal are aligned sequentially with the LOs. A polarization controller is required in one of the delayed paths, to align the polarizations of the two LO signals in the two consecutive time slots. The decomposition of the wide-band optical signal into two sub-band signals is achieved by the limited bandwidth of the coherent receivers, which is also referred as an analysis analog filter [2].

\section{Frequency stitching methods}

The algorithms for spectrally stitching the sliced signals are shown in Fig. 1 (b) and (c). After correcting the frontend impairments, the spectrally sliced sub-band of a single 60 GBaud Nyquist signal in the two time slots (see Fig. 2 (a)) are converted into parallel (see Fig. 2 (b)). The starting points in the two time slots needs to be calibrated by using a cross correlation between the received and the transmitted signal. Once the correct delay is found, the residual delay that is due to the environmental variation in the experiment can be digitally corrected by the stitching method. A digital filter $(H(\omega))$ can be applied to both parallel signals to reduce the out of band noise [2]. Here, we applied a digital 5th order Butterworth finite impulse response (FIR) filter with an optimum bandwidth of approximately $20 \mathrm{GHz}$. Both the higher (I) and lower (II) halves of the wideband signal are then upsampled to 160GS/s in the frequency domain and then frequency-translated back to their corresponding frequency by $\pm 15 \mathrm{GHz}$ respectively (see Fig. 2 (c)).

For phase based stitching, the signals within a bandwidth of approximately $5 \mathrm{GHz}$ around DC in both the higher and lower half of the wideband signal are filtered out to estimate the difference between the captured higher and lower sub-bands. First, the residual delay between them is corrected by using a cross correlation between the overlapped samples. After the delay correction, the phase difference is estimated by calculating the mean value between each samples within a block of $2^{\wedge} 17$ samples. By fitting the phase difference from the total 30 blocks linearly, the frequency deviation of the comb source can be corrected $( \pm 20 \mathrm{kHz}$ between adjacent comb lines), which is critical for the phase stitching method. Next, the two sub-band signals with the corrected phase and delay are coherently summed (see Fig. 2 (d)). Conventional DSP for a polarization multiplexed Nyquist signal is then applied with a 512 taps $2 \times 2$ frequency domain equalizer, first running in data-aided (DA) mode and then switched to decision-directed (DD) mode after convergence.

A $4 \times 2$ MIMO equalizer can also be used to stitch the two sub-bands together as shown in Fig. 1 (c). The four parallel inputs to the equalizer correspond to the two polarizations of the higher and lower frequency sub-bands of the wideband signal, which are frequency-translated to the corresponding frequencies (see Fig. 2 (c)). The equalizer can then adaptively estimate the phase difference as well as the amplitude and the residual delay mismatch between the two sub-bands and also apply a suppression of the frequency components in the overlapped region. Both the constant modulus algorithm (CMA) and the least mean square (LMS) algorithm are investigated and same performance is achieved for the QPSK signal while for the 64 quadrature amplitude modulation (QAM) signal, the DA-DD LMS algorithm is found to suffer from cycle slips. The received constellation diagram after the MIMO equalizer is shown in Fig. 1 (d), where DA-DD LMS algorithm is used for the QPSK signal whilst the CMA algorithm is used for the 64 QAM signal.

\section{Results}

The FS receiver is first employed to detect the Nyquist-shaped 60 GBaud DP-QPSK signal using a $20 \mathrm{GHz}$ receiver and ADCs sampled at $80 \mathrm{Gs} / \mathrm{s}$ with an LO spacing of $30 \mathrm{GHz}$. Fig. 4 shows the system performance in terms of BER as a function of received optical signal to noise ratio (OSNR). In Fig. 4 (a), the back to back performance of the two 

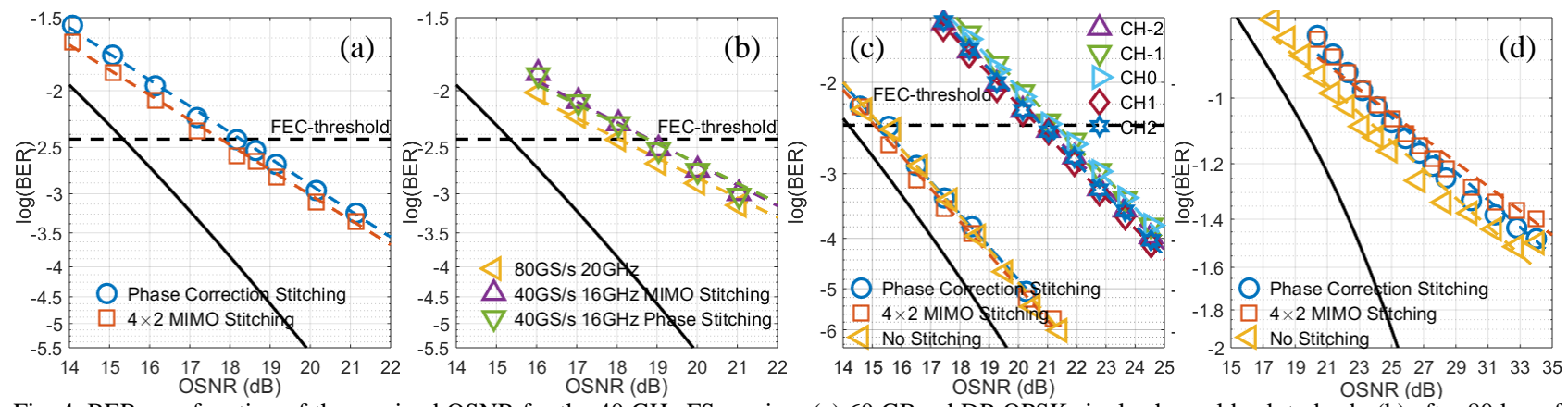

Fig. 4. BER as a function of the received OSNR for the $40 \mathrm{GHz}$ FS receiver (a) 60 GBaud DP-QPSK single channel back to back, (b) after $80 \mathrm{~km}$ of transmission. $246 \mathrm{GHz}$ FS receiver (c) 46 GBaud DP-QPSK single channel and 5 super-channels, and (d) 46 GBaud DP-64QAM single channel back to back.

stitching methods is compared and similar performance is observed. The wideband signal is then transmitted through an $80 \mathrm{~km}$ of standard single mode fiber (SMF), the performance is shown in Fig. 4(b). The fiber dispersion is compensated digitally after the phase correction. While, for the MIMO equalizer stitching methods, the dispersion compensation is applied individually on the four inputs to the MIMO equalizer. There is a $0.2 \mathrm{~dB}$ penalty comparing to the back to back case which shows that the dispersion of the fiber has little effect on the FS receiver. For a 60 Gbaud Nyquist signal, if two FS sub-bands are used, ideally a receiver with an electrical bandwidth of $15 \mathrm{GHz}$ is sufficient. In order to emulate these narrower bandwidth and lower speed ADCs, we digitally applied a $16 \mathrm{GHz}$ low pass FIR filter and afterwards downsampled the signal to $40 \mathrm{GS} / \mathrm{s}$ with a $2 \mathrm{GHz}$ overlap between the higher and lower subbands. The results are also displayed in Fig 4 (b), where a penalty of $0.7 \mathrm{~dB}$ is found for the lower sampling rate and bandwidth receiver. This penalty is due to trade-off between the roll-off of the FIR filter and the aliasing.

The FS receiver can also be used to coherently detect ultra-wideband super-channels as this enables digital back propagation of the signal for both linear and nonlinear distortion mitigation. In order to demonstrate the capability of receiving an ultra-wideband super-channel, a $5 \times 46$ GBaud Nyquist DP signal is generated with two IQ-modulators for the odd and even channels respectively. The channel spacing is set at $49 \mathrm{GHz}$. A dual polarization coherent receiver (DP-CoRx) with an electrical bandwidth of $63 \mathrm{GHz}$ is used in the FS receiver to achieve a stitched optical bandwidth of $246 \mathrm{GHz}$ with a $6 \mathrm{GHz}$ overlap between the lower and higher sub-bands. The comb spacing is set at $20 \mathrm{GHz}$ and two comb lines separated by $120 \mathrm{GHz}$ are selected as the LOs. The stitched spectrum of the super-channel is shown in Fig. 2 (e). where the central channel is split over the two sub-bands. To validate the performance of the stitching technique we first captured a single $46 \mathrm{Gbaud}$ channel using a single $\mathrm{LO}$ without frequency stitching, and then captured the same signal using the FS receiver with the channel aligned such that it is split over the two sub-bands. As shown in Fig. 4 (c) there is no penalty incurred when using the stitched receiver. Also shown is the BER performance using the phase based stitching when all 5 sub-channels are present where the variation in OSNR penalty between the five sub-channels is found to be less than $0.6 \mathrm{~dB}$. There is a $5.8 \mathrm{~dB}$ performance penalty between super-channel and single channel operation arising from the limited common mode rejection ratio of the receiver, where the sub-band that is outside the receiver bandwidth contributes to an unwanted directly detected signal. For the DP-64 QAM signal, it was not possible to use the DA-DD LMS MIMO equalizer due to cycle slips in the phase estimation and a blind CMA equalizer was used which resulted in a $1.7 \mathrm{~dB}$ penalty between the stitched and non-stitched scenarios (see Fig. 4 (d)).

\section{Conclusion}

We demonstrate a time multiplexed FS receiver that can increase the bandwidth of optical coherent receivers beyond the electronic bandwidth limits using only a single coherent receiver. We demonstrate the detection of a 60 GBaud DP Nyquist QPSK signal using a single $16 \mathrm{GHz}$ bandwidth receiver with $40 \mathrm{GS} / \mathrm{s}$ ADCs and simultaneous reception of 5×46 GBaud DP Nyquist QPSK and DP-64 QAM super-channels, occupying $246 \mathrm{GHz}$, using a single DP-CoRx with an electrical bandwidth of $63 \mathrm{GHz}$.

This work was supported by UK EPSRC funding through project EP/J008842/1

\section{References}

[1] N. K. Fontaine et al., "Fiber nonlinearity compensation by digital backpropagation of an entire 1.2-Tb/s superchannel using a full-field spectrally-sliced receiver," ECOC 2013, Mo.3.D.5, 2013.

[2] S. J. Mazlouman, S. Sheikhaei and S. Mirabbasi, "Digital Compensation Techniques for Frequency-Translating Hybrid Analog-to-Digital Converters," IEEE Transactions on Instrumentation and Measurement, vol. 60, no. 3, pp. 758-767, 2011.

[3] N. K. Fontaine et al., "228-GHz Coherent Receiver using Digital Optical Bandwidth Interleaving and Reception of 214-GBd (856-Gb/s) PDM-QPSK," ECOC, Th.3.A, 2012.

[4] Roy G. H. van Uden et al., "Time domain multiplexed spatial division multiplexing receiver," Optics Express, vol. 22, no. 10, 2014 [5] Masaki Sato et al, "Frequency Diversity MIMO Detection for DP- QAM Transmission," J. Lightwave Technol. 33, 1388-1394 (2015). [6] Rui Wu et al., "Generation of very flat optical frequency combs from continuous-wave lasers using cascaded intensity and phase modulators driven by tailored radio frequency waveforms," Opt. Lett., vol. 35, no. 19, pp. 3234-3236, 2010. 